\title{
Necessary Conditions for Apparent Horizons and Singularities in Spherically Symmetric Initial Data
}

\author{
Jemal Guven ${ }^{(1)} *$ and Niall Ó Murchadha ${ }^{(2)} \dagger$ \\ (1) Instituto de Ciencias Nucleares \\ Universidad Nacional Autónoma de México \\ Apdo. Postal 70-543, 04510 México, D.F., MEXICO \\ (2) Physics Department, University College Cork \\ Cork, IRELAND
}

\begin{abstract}
We establish necessary conditions for the appearance of both apparent horizons and singularities in the initial data of spherically symmetric general relativity when spacetime is foliated extrinsically. When the dominant energy condition is satisfied these conditions assume a particularly simple form. Let $\rho_{\text {Max }}$ be the maximum value of the energy density and $\ell$ the radial measure of its support. If $\rho_{\mathrm{Max}} \ell^{2}$ is bounded from above by some numerical constant, the initial data cannot possess an apparent horizon. This constant does not depend sensitively on the gauge. An analogous inequality is obtained for singularities with some larger constant. The derivation exploits Poincaré type inequalities to bound integrals over certain spatial scalars. A novel approach to the construction of analogous necessary conditions for general initial data is suggested.
\end{abstract}

* jemal@nuclecu. unam.mx

$\dagger$ niall@ucc.ie 


\section{INTRODUCTION}

In this paper we cast necessary conditions for the appearance of apparent horizons and singularities in the initial data of spherically symmetric general relativity. This is the natural sequel to a previous paper in which we examined sufficiency conditions in the same context (1] [2]. The formulation of necessary conditions is clearly more difficult. This is because by the nature of the problem, we must assume the worst: a scenario in which the geometry possesses an apparent horizon or a singularity.

The initial data consists of the intrinsic and extrinsic geometry on some spacelike hypersurface. We suppose that the line element on the spatial geometry is parametrized by

$$
d s^{2}=d \ell^{2}+R^{2} d \Omega^{2}
$$

Here $\ell$ is the radial length on the surface. $4 \pi R^{2}$ is the area of a sphere of fixed $\ell$. We can express the spherically symmetric extrinsic curvature in terms of two spatial scalars, $K_{\mathcal{L}}$ and $K_{R}$

$$
K_{a b}=n_{a} n_{b} K_{\mathcal{L}}+\left(g_{a b}-n_{a} n_{b}\right) K_{R}
$$

Here $n^{a}$ is the outward pointing unit normal to the two-sphere of fixed radius. We have that $R, K_{R}$ and $K_{\mathcal{L}}$ are constrained by the hamiltonian and momentum constraints,

$$
\frac{1}{2}\left(1+R^{\prime 2}\right)-\left(R R^{\prime}\right)^{\prime}=4 \pi \rho R^{2}+\frac{1}{2} R^{2}\left(2 K_{\mathcal{L}}-K_{R}\right) K_{R}
$$

and

$$
K_{R}^{\prime}+\frac{R^{\prime}}{R}\left(K_{R}-K_{\mathcal{L}}\right)=4 \pi J
$$

The primes represent derivatives with respect to $\ell$. We assume that both the energy density of the matter $\rho$ and its current $J$ are finite.

We exploit an extrinsic time foliation. This involves a constraint on the two extrinsic curvature scalars. We will suppose that this constraint is quasi - linear, and homogeneous so that

$$
K_{\mathcal{L}}+\alpha K_{R}=0
$$

where $\alpha$ is some specified not necessarily local function of the configuration variables, $\alpha=$ $\alpha\left(K_{R}, R, \ell\right)$ which is bounded from below by 0.5 [3. 4 .

By a regular geometry, in this paper we understand any spatial geometry with a single asymptotically flat region and a regular center, $\ell=0$, without either apparent horizons or singularities. The appropriate boundary condition on the metric at $\ell=0$ is then

$$
R(0)=0
$$

The occurence of apparent horizons or singularities in spherically symmetric general

relativity is due entirely to the action of matter. Though $\rho$ and $J$ are finite there is no guarantee that a regular asymptotically flat solution defined for all $\ell \geq 0$ will exist [4]. 
At a future (past) apparent horizon, the optical scalar $\omega_{ \pm}$defined by [5] [3, (1]

$$
\omega_{ \pm}=2\left(R^{\prime} \pm R K_{R}\right)
$$

vanishes,

$$
\omega_{ \pm}\left(\ell_{H}\right)=0 .
$$

To avoid clutter we will focus only on future horizons in this paper.

Singularities occur when the geometry pinches off at some finite proper radius, $\ell_{S}$, from the center,

$$
R\left(\ell_{S}\right)=0 .
$$

A singular geometry necessarily contains at least one kind of apparent horizon. If the massfunction becomes negative as one approaches the singularity one must have both future and past horizons.

To provide necessary conditions for an apparent horizon or a singularity we consider the bounded region enclosed by the feature in question. The boundary condition (8) or (9) is then imposed on Eqs.(3) and (44). Integrating Eq.(3) over the domain $\left[0, \ell_{H}\right]$ or $\left[0, \ell_{S}\right]$ then provides an integrability condition on the spatial geometry and the sources. This integrability condition then provides the basis for an inequality bounding some measure of the energy content of the region by some measure of its size.

What constitutes a natural measure of the energy content is a subtle issue. In our examination of sufficiency conditions we found that the appropriate measures were the total enclosed material energy, $M$, or the difference, $M-P$, where $P$ is the material current [1]. We found that if either the weak or the dominant energy condition holds, and the geometry did not possess an apparent horizon, then $M-P<$ constant $\ell$, where $\ell$ is the radial support, for some constant of order unity [国]. The same inequality with $M-P$ replaced by $M$ and with some larger constant is obtained for singularities.

In [6], where we addressed the problem when the geometry is momentarily static, we saw that $M$ can remain small though $\ell$ be arbitrarily large. This can occur because $R$ is folded into the definition of $M$ and $R$ can either saturate or worse become small. One should not therefore expect $M$ to serve as a useful measure of the material energy for the purpose of casting necessary conditions. Indeed, we know that the statement: if $M \leq$ constant $\ell$ then the geometry is non - singular - cannot be justified [7].

In [6], however, with $K_{a b}=0$ we did identify variables with respect to which non-trivial necessary conditions could be cast of the form: if

$$
\rho_{\text {Max }} \ell^{2}<\text { constant },
$$

where $\rho_{\mathrm{Max}}$ is the maximum energy density, the distribution of matter will not possess an apparent horizon with one constant; with some larger constant it will not possess a singularity.

When matter flows, the obvious generalization of $\rho_{\mathrm{Max}}$ is the sum $\rho_{\mathrm{Max}}+J_{\mathrm{Max}}-$ however, $\rho_{\text {Max }}$ and $J_{\text {Max }}$ do not enter symmetrically into the inequality. Unlike the sufficiency conditions which involved a symmetric combination of $M$ and $P$, the equalities we obtain 
do not respect this symmetry. The natural inequality we obtain involves not only $J$ but its square, assuming the form: if

$$
\rho_{\mathrm{Max}} \ell^{2}+c_{0} J_{\mathrm{Max}} \ell^{2}+c_{1}\left(J_{\mathrm{Max}} \ell^{2}\right)^{2}<c_{2}
$$

where $c_{0}, c_{1}$ and $c_{2}$ are three given numerical constants, then the geometry is regular. Once a choice of gauge has been made the symmetry between $\rho$ and $J$ is necessarily broken. Despite appearances this is not an artifact of the extrinsic time slicing we have exploited. The value of $J$ plays a more significant role than the value of $\rho$. This is consistent with our findings in [4] in our examination of the generic behavior of the metric in the neighborhood of a singularity in an $\alpha$-foliation of spacetime.

When the dominant energy condition,

$$
\rho \geq|J|
$$

is satisfied, the momentarily static form (10) obtains from Eq.(11) with some larger constant which depends only weakly on $\alpha$. This is remarkable in many ways. The single hamiltonian constraint is replaced by the two coupled equations, (3) and (4) satisfying the gauge condition, (5); in the worst scenario we must assume, not only do we need to contend with potential divergences in the intrinsic geometry but, in addition, with divergences in the extrinsic curvature.

The paper is organized as follows. In Sect.2, we collect some relevant bounds on potential divergences. In Sect.3, we discuss the weights which must be introduced into integrals over relevant geometrical scalars to render them well defined when the scalar is singular at the end point of the domain of integration. In Sect.4, we derive a necessary condition of the form (11) for singularities. In Sect.5, we do the same for apparent horizons. In Sect.6, we derive a simple necessary condition for the appearance of minimal surfaces. We end with brief discussion. Derivations of mathematical inequalities are provided in an appendix.

\section{BOUNDS ON $R, R^{\prime}$ AND $K_{R}$}

To formulate a necessary condition for singularities it is important to possess some bound limiting the maximum values of $R^{\prime}$ and $K_{R}$ which does not require the geometry to be regular. In particular, one cannot exploit the numerical bounds on these variables derived in [4] which rely on the regularity of the geometry. Indeed these quantities can be arbitrarily large. What we need to do is place an upper bound on their rate of divergence in the neighborhood of singularities. These bounds will then be applied to determine the weights which are appropriate to turn the integrability condition into an inequality. In fact, this will be their only use in this paper.

We first recall that Eq.(1) can be solved for $K_{R}$ in terms of the radial flow of matter, $J$, as follows

$$
K_{R}=\frac{4 \pi}{R^{1+\alpha}} \int_{0}^{\ell} d \ell_{1} R^{1+\alpha} J \Delta\left(\ell_{1}, \ell\right)
$$

The positive factor $\Delta$ is given by 


$$
\Delta\left(\ell_{1}, \ell\right)=e^{\int_{\ell_{1}}^{\ell} d \ell_{2} \alpha^{\prime} \ln (R / L)},
$$

where $L$ is some arbitrary length scale. If $\alpha$ is constant, $\Delta=1$. This form of the solution makes explicit the fact that spatial variations of $\alpha$ can be absorbed into a multiplicative dressing of the current density. The constant $\alpha$ result is modulated by $\Delta$.

It is now straightforward to place a bound on $K_{R}$. We have

$$
K_{R} \leq \frac{4 \pi\left|J_{\mathrm{Max}}\right|}{R^{1+\alpha}} \Delta(0, \ell) \int_{0}^{\ell} d \ell_{1} R^{1+\alpha} .
$$

We saw in 4 that $\Delta$ is, in fact, finite everywhere. It is possible to further bound $\Delta(0, \ell)$ by bounding $\alpha^{\prime}$ by $\left|\alpha^{\prime}\right|_{\text {Max }}$ and pulling it through the integral. However, we will treat the integral appearing in the exponent itself as the natural measure of the variation of $\alpha$. Just as we found that $\alpha \geq 0.5$, we will need to bound the variation of $\alpha$ appropriately if we are not to be overwhelmed by gauge introduced noise in casting necessary conditions. Recall that no such bound was ever invoked when we addressed sufficiency conditions in [1].

The exact expression (13) and the bound (15) determines the potential divergence of $K_{R}$ at a singularity. This occurs with $R$ returning to zero at some finite radius from the center, at $\ell=\ell_{S}$. In [4], we saw that in the neighborhood of this point

$$
R \sim\left(\frac{C_{\alpha}}{\alpha+1}\right)^{\frac{1}{\alpha+1}}\left(\ell_{S}-\ell\right)^{\frac{1}{\alpha+1}},
$$

where $C_{\alpha}$ is the finite constant,

$$
C_{\alpha}=\int_{0}^{\ell_{S}} d \ell_{1} R^{1+\alpha} J \Delta\left(\ell_{1}, \ell\right) .
$$

Generically, therefore, $R^{\prime}$ diverges at $\ell_{S}$ as do all higher derivatives of $R$. If $\alpha\left(\ell_{S}\right)>0.5$, such spatial singularities are more severe than the strong singularities discussed in [6] which are consistent with the Hamiltonian constraint at a moment of time symmetry. Increasing this value of $\alpha$ increases the strength of the singularity.

Even if the geometry is singular so that $R^{\prime}$ diverges, it can only diverge to minus infinity - the surface $R^{\prime}=1$ in the configuration space can never be breached from below. We always have $R^{\prime} \leq 1$ 近.

\section{POINCARÉ INEQUALITIES, WEIGHTS AND MEASURES}

Crucial to the derivation of Eq.(10) in [6] were two simple Poincaré inequalities of the form

$$
S \int_{0}^{\ell_{1}} d \ell R^{2} \leq \int_{0}^{\ell_{1}} d \ell R^{\prime 2},
$$

where $S$ depends on the boundary conditions satisfied by $R$. In general $R(0)=0$. At the first trapped surface, $R^{\prime}\left(\ell_{1}\right)=0$ and $S=\pi^{2} / 4 \ell_{1}^{2}$. At a singularity, $R\left(\ell_{1}\right)=0$ and $S=\pi^{2} / \ell_{1}^{2}$.

Recall that because $R^{\prime} \leq 1, R$ is always bounded by $\ell$. This guarantees that if the geometry is small in the radial direction it will also be small in the two transverse directions. 
A consequence is that any integral over a positive powers of $R$ will be well defined over any finite interval. At a singularity, in a moment-of-time-symmetry slice, however, we found that $R$ tends to zero like $R \sim\left(\ell_{S}-\ell\right)^{2 / 3}$ so that $R^{\prime}$ diverges like $\left(\ell_{S}-\ell\right)^{-1 / 3}$. Even though $R^{\prime}$ diverges so that the integrand on the RHS of Eq.(18) diverges, the integral itself remains finite. When $J \neq 0, R^{\prime}$ can diverge more rapidly. Eq.(16) implies $R^{\prime} \sim\left(\ell_{S}-\ell\right)^{-\alpha / 1+\alpha}$. Thus the integral on the RHS of Eq.(18) will only exist if $\alpha<1$. This is outside the range found to provide the best sufficiency results in [1]. To remedy this situation a non-trivial weight function will need to be introduced into the integrand to render the bounding integral well defined. In [6], we found that we could improve the inequalities of necessity at a moment of time symmetry by weighting with an appropriate power of $R$. Here it will be essential.

Again, let this function be some power of $R, R^{a}$ say. The relevant exponent will generally depend on $\alpha$. At a singularity, $R^{a} R^{\prime 2} \sim\left(\ell_{S}-\ell\right)^{(a-2 \alpha) /(1+\alpha)}$. The integral

$$
\int_{0}^{\ell_{1}} d \ell R^{a} R^{\prime 2}
$$

will exist for all $a>\alpha-1$. This is not, however, the optimal value for our purposes. We will see below that a larger value is desirable. If $a$ is constant, we have $R^{a / 2} R^{\prime}=$ $\left(R^{1+a / 2}\right)^{\prime} /(1+a / 2)$. We then simply apply Eq.(18) to the function $R^{1+a / 2}$ in place of $R$.

\section{SINGULARITIES}

When the gauge condition, Eq.(5) is satisfied, we note that the Hamiltonian constraint assumes the form

$$
\frac{1}{2}\left(1+R^{\prime 2}\right)=\left(R R^{\prime}\right)^{\prime}+4 \pi \rho R^{2}+\frac{1}{2}(2 \alpha-1) R^{2} K_{R}^{2} .
$$

The second and third terms on the RHS are manifestly positive. Suppose that the geometry is singular at $\ell=\ell_{S}$. We cannot simply integrate Eq.(20) and discard the boundary term. First of all, as we pointed out above, it is clear from Eq. (16) that the integral of $R^{\prime 2}$ does not exist on the interval $\left[0, \ell_{S}\right]$; in addition, the surface term $R R^{\prime}$ does not vanish at the singularity unless $\alpha<1$ there. To remedy the problem we multiply Eq.(20), as discussed in Sect.3, by an appropriate weight function, $R^{a}$, before integration.

This multiplication has the unfortunate side - effect of destroying the divergence $\left(R R^{\prime}\right)^{\prime}$ appearing on the RHS of Eq.(20). It is, however, simple to restore this divergence: we note that

$$
\left(R^{1+b}\right)^{\prime}=(1+b) R^{b} R^{\prime}-b^{\prime} R^{1+b} \ln R / \ell_{S} .
$$

We perform an integration by parts on the term $R^{a}\left(R R^{\prime}\right)^{\prime}$, and now substitute the RHS of Eq.(21) for $\left(R^{a}\right)^{\prime}(a=b+1)$ :

$$
\begin{aligned}
\frac{1}{2} \int_{0}^{\ell_{S}} d \ell R^{a}\left(1+(2 a+1) R^{2}\right)= & \left.R^{1+a} R^{\prime}\right|_{\ell_{S}}+4 \pi \int_{0}^{\ell_{S}} d \ell \rho R^{2+a}-\Phi \\
& +\frac{1}{2} \int_{0}^{\ell_{1}} d \ell(2 \alpha-1) R^{2+a} K_{R}^{2},
\end{aligned}
$$


where $\Phi$, given by

$$
\Phi=\int_{0}^{\ell_{S}} d \ell a^{\prime} \ln \left(R / \ell_{S}\right) R^{1+a} R^{\prime}
$$

is a correction which vanishes if $\alpha^{\prime}=0$. To discard the boundary term, we require $R^{1+a} R^{\prime}$ to vanish at the singularity. This implies that

$$
a>\alpha-1 .
$$

This choice of a simultaneously bounds the integral over $R^{a} R^{\prime 2}$.

We also will need to place a bound on the last term on the RHS of Eq.(22). We exploit Eq.(15) to bound $K_{R}$. The problem is that this bound involves the positive power of $R$, $R^{1+\alpha}$, in the denominator which is difficult to control. We obtain the bound,

$$
\int_{0}^{\ell_{S}} d \ell(2 \alpha-1) R^{2+a} K_{R}^{2} \leq(4 \pi)^{2}\left(2 \alpha_{\mathrm{Max}}-1\right) J_{\mathrm{Max}}^{2} \Delta\left(0, \ell_{S}\right)^{2} \int_{0}^{\ell_{S}} d \ell R^{a-2 \alpha}\left(\int_{0}^{\ell} d \ell R^{1+\alpha}\right)^{2}
$$

on this term. If the weighting exponent is chosen such that

$$
a \geq 2 \alpha
$$

the denominator is removed by the weight. Fortunately, such values are consistent with Eq.(24) for all physically acceptable values of $\alpha$. The RHS of Eq.(22) is clearly simplest when

$$
a=2 \alpha .
$$

This is the value we will henceforth adopt for $a$.

The expression on the right hand side of Eq.(25) is still not very useful as it stands. A remarkable fact, however, is that we can bound it by an integral over $R^{2(1+\alpha)}$. In fact, we have the following inequality

$$
\int_{0}^{\ell_{1}} d \ell\left(\int_{0}^{\ell} d \ell R^{1+\alpha}\right)^{2} \leq\left(\frac{2}{\pi}\right)^{2} \ell_{1}^{2} \int_{0}^{\ell_{1}} d \ell R^{2(\alpha+1)} .
$$

This result is derived in the appendix. Eq.(28) implies the bound for the $K_{R}^{2}$ term:

$$
\int_{0}^{\ell_{S}} d \ell(2 \alpha-1) R^{2(1+\alpha)} K_{R}^{2} \leq 64\left(2 \alpha_{\mathrm{Max}}-1\right) J_{\operatorname{Max}}^{2} \Delta\left(0, \ell_{S}\right)^{2} \ell_{S}^{2} \int_{0}^{\ell_{S}} d \ell R^{2(1+\alpha)} .
$$

To understand why this bound is important, note that we can exploit the identity (21) to cast the integrand $R^{2 \alpha} R^{\prime 2}$ appearing on the LHS of Eq.(22) in the form

$$
R^{2 \alpha} R^{\prime 2}=\frac{1}{(1+\alpha)^{2}}\left(\left(R^{1+\alpha}\right)^{\prime 2}-\alpha^{\prime 2} R^{2+2 \alpha} \ln ^{2}\left(R / \ell_{S}\right)\right)-\frac{2 \alpha^{\prime}}{1+\alpha} R^{1+2 \alpha} R^{\prime} \ln \left(R / \ell_{S}\right) .
$$

If $\alpha$ is constant only the first term survives. Let us focus on this term. A one-dimensional Poincaré inequality can be exploited to place a lower bound on the integral over $\left(R^{(1+\alpha)}\right)^{\prime 2}$ : 


$$
S \int_{0}^{\ell_{1}} d \ell R^{2(1+\alpha)} \leq \int_{0}^{\ell_{1}} d \ell\left(R^{1+\alpha}\right)^{\prime 2}
$$

where the constant $S=\pi^{2} / \ell_{1}^{2}$ is the constant which is relevant for functions which vanish at both $\ell=0$ and $\ell=\ell_{1}$.

If $\alpha$ is constant, we then have

$$
\int_{0}^{\ell_{S}} d \ell R^{2 \alpha} \leq 2\left[4 \pi \rho_{\mathrm{Max}}+32(2 \alpha-1) J_{\mathrm{Max}}^{2} \ell_{S}^{2}-\left(\frac{\pi}{\ell_{S}}\right)^{2} \frac{1+4 \alpha}{2(1+\alpha)^{2}}\right] \int_{0}^{\ell_{S}} d \ell R^{2(1+\alpha)} .
$$

In [6], we proved that when Eq.(6) is satisfied and $R^{\prime} \leq 1$ then the ratio of the integrals appearing in Eq.(32) can be bounded as follows (Eq.(6.3.16)) $(a=2 \alpha)$

$$
\frac{\int_{0}^{\ell_{1}} R^{2+a} d \ell}{\int_{0}^{\ell_{1}} R^{a} d \ell} \leq \frac{1+a}{3+a} \ell_{1}^{2}
$$

Eq.(33) implies

$$
\frac{1}{2} \frac{3+2 \alpha}{1+2 \alpha}+\frac{1+4 \alpha}{2(1+\alpha)^{2}} \pi^{2} \leq 4 \pi \rho_{\operatorname{Max}} \ell_{1}^{2}+32(2 \alpha-1) J_{\mathrm{Max}}^{2} \ell_{1}^{4}
$$

We note that it is the second term on the left hand side which will determine the bound for $\alpha \sim+1$. It is maximized when $\alpha=0.5$. With this value, we reproduce the moment of time symmetry result [6] - this is a peculiarity of this gauge.

The dependence on the value of $\alpha$ will generally not be a strong one so long as $\alpha$ is bounded. In particular, if $\alpha=1$

$$
\frac{5 \pi}{32}\left[1+\frac{4}{3 \pi^{2}}\right] \leq \rho_{\mathrm{Max}} \ell_{1}^{2}+\frac{8}{\pi}\left(J_{\mathrm{Max}} \ell_{1}^{2}\right)^{2} .
$$

Note the asymmetry between the roles of $\rho_{\text {Max }}$ and $J_{\text {Max }}$. The inequality does not involve what one would to be the obvious generalization of $\rho_{\text {Max }}$, the sum $\rho_{\text {Max }}+J_{\text {Max }}$. $J_{\text {Max }}$ plays a more decisive role than $\rho_{\text {Max }}$ in the inequality, appearing as it does through its square in contrast to $\rho$ which appears linearly. The inequality with $K_{a b}=0$ does not generalize in the obvious linear way. If the dominant energy condition Eq.(12) holds, the inequality simplifies. For $\alpha=1$ we obtain

$$
\frac{1}{8}\left[\sqrt{\frac{5}{3}+\frac{3}{2} \pi^{2}}-\frac{\pi}{2}\right] \leq \rho_{\operatorname{Max}} \ell_{S}^{2}
$$

The LHS $\sim 5 / 16$, which is approximately half as good as the moment of time symmetry result.

If $\alpha$ is not a constant, additional noise is introduced into the inequality by the gauge. We get

$$
\int_{0}^{\ell_{1}} d \ell R^{2 \alpha} \leq 2\left[4 \pi \rho_{\operatorname{Max}}+32\left(2 \alpha_{\operatorname{Max}}-1\right) J_{\operatorname{Max}}^{2} \Delta^{2} \ell_{1}^{2}-\left(\frac{\pi}{\ell_{1}}\right)^{2} \frac{1+4 \alpha}{2(1+\alpha)^{2}}\right] \int_{0}^{\ell_{1}} d \ell R^{2(1+\alpha)}+\Phi_{1}+\Phi_{2},
$$


where $\Phi_{1}$ and $\Phi_{2}$ are given respectively by

$$
\Phi_{1}=\int_{0}^{\ell_{1}} d \ell \frac{2 \alpha-1}{1+\alpha} \alpha^{\prime} \ln R / \ell_{1} R^{1+2 \alpha} R^{\prime}
$$

and

$$
\Phi_{2}=\int_{0}^{\ell_{1}} d \ell \frac{1+4 \alpha}{2(1+\alpha)^{2}} \alpha^{2} \ln ^{2} R / \ell_{1} R^{2(1+\alpha)} .
$$

The spatial dependence of $\alpha$ is encoded in $\Delta$ and two terms $\Phi_{1}, \Phi_{2}$ which get picked up in the trade off of $R^{\alpha} R^{\prime}$ for $\left(R^{1+\alpha}\right)^{\prime}$. $\Phi_{1}$ includes the contribution from $\Phi$ appearing in Eq.(22).

These integrals can both be bounded. We have

$$
\Phi_{1} \leq \frac{2 \alpha_{\mathrm{Max}}-1}{1+\alpha_{\mathrm{Min}}} R_{\mathrm{Max}}^{1+2 \alpha} \int_{0}^{R_{\mathrm{Max}}} d R\left|\alpha^{\prime}\right|\left|\ln R / \ell_{1}\right|
$$

and

$$
\Phi_{2} \leq \frac{1+4 \alpha_{\mathrm{Max}}}{2\left(1+\alpha_{\mathrm{Min}}\right)^{2}} R_{\mathrm{Max}}^{2(1+\alpha)} \int_{0}^{\ell_{1}} d \ell \alpha^{\prime 2} \ln ^{2} R / \ell_{1} .
$$

The integrated logarithm appearing in Eq.(40) is bounded by that which appears in the definition, Eq.(14) of $\Delta$. Clearly, we can bound both by (the square root of) the integral appearing in Eq.(41). This is the only measure of $\alpha^{\prime}$ we need to control. We will also need the bounds

$$
R_{\mathrm{Max}}^{n+2 \alpha} / \int_{0}^{\ell_{1}} d \ell R^{2 \alpha} \leq\left(1+2 \alpha_{\mathrm{Max}}\right) \ell_{1}^{n-1}
$$

for $n \geq 1$.

\section{APPARENT HORIZONS}

At a moment of time symmetry, there is a remarkable similarity between the signal for the presence of an apparent horizon, $R^{\prime}=0$ and that for the presence of a singularity, $R=0$. In [6], this meant that the techniques we exploited for analysing singularities were also good for analyzing apparent horizons and the effort required almost identical. In general, however, the signal for an apparent horizon will involve the extrinsic curvature of the spatial hypersurface through Eq.(8). Its physical location no longer coincides with an extremal surface of the spatial geometry as it did at a moment of time symmetry.

At a future apparent horizon, $\omega_{+}$defined by Eq.(7) vanishes. Eliminating $R^{\prime}$ in the divergence term in Eq.(3) using Eq.([) we obtain

$$
\frac{1}{2}\left(1+R^{\prime 2}\right)=\left(R \omega_{+}-R^{2} K_{R}\right)^{\prime}+4 \pi \rho R^{2}+\frac{1}{2}(2 \alpha-1) R^{2} K_{R}^{2} .
$$

Again both the second and third terms on the RHS are manifestly positive. Let us suppose for simplicity that $\alpha$ is constant. 
Suppose that all quantities are well defined (we will relax this assumption below). We can then integrate Eq.(43) up to the first future horizon at which $\omega_{+}=0$ to get

$$
\int_{0}^{\ell_{1}} d \ell\left(1+R^{\prime 2}\right)=-\left.R^{2} K_{R}\right|_{\ell_{1}}+4 \pi \int_{0}^{\ell_{1}} d \ell R^{2} \rho+\frac{1}{2}(2 \alpha-1) \int_{0}^{\ell_{1}} d \ell R^{2} K_{R}^{2}
$$

We wish to exploit Eq.(15) to place a bound on $K_{R}$ in the surface term. Unfortunately, this bound will only be well defined for $\alpha \leq 1$.

The first two terms can be dealt with symmetrically when $\alpha=1$. In this case these first two terms on the RHS can be bounded as follows:

$$
-\left.R^{2} K_{R}\right|_{\ell_{1}}+4 \pi \int_{0}^{\ell_{1}} d \ell R^{2} \rho \leq 4 \pi\left(\rho_{M a x}+\left|J_{M a x}\right|\right) \int_{0}^{\ell_{1}} d \ell R^{2} .
$$

A linear term in $J_{\text {Max }}$ appears in the apparent horizon inequality condition which is not present in the singularity inequality. This is a reflection of the different boundary conditions enforced there.

We can exploit a Poincaré inequality to place a bound on the integral over the interval $\left[0, \ell_{1}\right]$ of the quadratic $R^{2}$ by the same integral over the quadratic, $R^{\prime 2}$ :

$$
S \int_{0}^{\ell_{1}} d \ell R^{2} \leq \int_{0}^{\ell_{1}} d \ell R^{\prime 2}
$$

The inequality is saturated by the trigonometric function,

$$
R(\ell)=\sin (\gamma \ell)
$$

which also determines the optimal value of $S=\gamma^{2}$. The boundary condition, (8) determines $\gamma$ to be the lowest solution of the transcendental equation,

$$
\tan \gamma \ell_{1}=-\frac{\gamma}{K_{R}}
$$

We note that

$$
\gamma \leq \frac{\pi}{2 \ell_{1}}
$$

if $K_{R}$ is negative with $\gamma \rightarrow \pi / 2 \ell_{1}$ as $K_{R} \rightarrow 0$ which is the moment of time symmetry bound and $\gamma \rightarrow \pi / \ell_{1}$ as $K_{R} \rightarrow+\infty$.

Unfortunately, even when $\alpha=1$, when we attempt to bound the third term on the right hand side we run into the same problem we faced when we examined singularities in Section 4 with the same term. We need to introduce a weighting to guarantee convergence of the integral. The same weighting which worked for singularities works again. There is no real simplification in the $\alpha=1$ case so we will return to the general case. To restore the divergence appearing in Eq.(43) we need to perform an integration by parts as before. We integrate up to $\ell_{1}$ :

$$
\begin{aligned}
\frac{1}{2} \int_{0}^{\ell_{1}} d \ell R^{2 \alpha}\left(1+(4 \alpha+1) R^{\prime 2}\right)= & -\left.R^{2(1+\alpha)} K_{R}\right|_{\ell_{1}}+4 \pi \int_{0}^{\ell_{1}} d \ell \rho R^{2(1+\alpha)} \\
& +\frac{1}{2}(2 \alpha-1) \int_{0}^{\ell_{1}} R^{2(1+\alpha)} K_{R}^{2}
\end{aligned}
$$


We now exploit Eq.(15) to bound the $K_{R}$ and $K_{R}^{2}$ terms. For the former,

$$
\left.R^{2(1+\alpha)} K_{R}\right|_{\ell_{1}} \leq 4 \pi R^{1+\alpha} J_{\operatorname{Max}} \int_{0}^{\ell_{1}} d \ell R^{1+\alpha}
$$

The weighting process has broken the symmetry under interchange of $\rho$ and $J$ of the linear terms on the RHS of Eq.(44) which is evident in Eq.(45). For the term quadratic in $K_{R}$, we again have $(29)$ with $a=2 \alpha)$

$$
\int_{0}^{\ell_{1}} d \ell R^{2(1+\alpha)} K_{R}^{2} \leq(4 \pi)^{2} J_{\operatorname{Max}}^{2} \int_{0}^{\ell_{1}} d \ell\left(\int_{0}^{\ell} d \ell R^{1+\alpha}\right)^{2} .
$$

We again require a bound on the last term by an integral over $R^{2(1+\alpha)}$. Though the boundary conditions are different we again obtain the bound (28). We demonstrate this in the appendix. We can now write

$$
\begin{array}{r}
1 \leq 2\left[4 \pi \rho_{\mathrm{Max}}+32(2 \alpha-1) J_{\operatorname{Max}}^{2} \ell_{1}^{2}-\tilde{\gamma}^{2} \frac{1+4 \alpha}{2(1+\alpha)^{2}}\right] \quad \int_{0}^{\ell_{1}} d \ell R^{2(1+\alpha)} / \int_{0}^{\ell_{1}} d \ell R^{2 \alpha} \\
+8 \pi J_{\operatorname{Max}} R_{1}^{1+\alpha} \int_{0}^{\ell_{1}} d \ell R^{1+\alpha} / \int_{0}^{\ell_{1}} d \ell R^{2 \alpha} .
\end{array}
$$

Here $\tilde{\gamma}$ is the analogue of the $\gamma$ that appears in Eqs.(47 - 48), except that $R^{2}$ in Eq.(46) is replaced by $R^{2(1+\alpha)}$. This means that Eq.(48) must be replaced by

$$
\tan \tilde{\gamma} \ell_{1}=-\frac{\tilde{\gamma}}{K_{R}(1+\alpha)}
$$

The same upper and lower bounds on $\tilde{\gamma}$ hold, i.e., $\pi / 2 \ell \leq \tilde{\gamma} \leq \pi / \ell_{1}$. We can again exploit (33) to bound the ratio of the integrals in the first term of (53). In the second term, one can exploit

$$
R_{1}^{1+\alpha} \int_{0}^{\ell_{1}} d \ell R^{1+\alpha} / \int_{0}^{\ell_{1}} d \ell R^{2 \alpha} \leq \frac{1+2 \alpha}{2+\alpha} \ell_{1}^{2}
$$

This is proved using the same technique as the derivation of Eq.(33). The necessary condition for an apparent horizon with constant $\alpha$ is then

$$
4 \pi\left(\rho_{\operatorname{Max}}+\frac{3+2 \alpha}{2+\alpha} J_{\text {Max }}\right) \ell_{1}^{2}+32(2 \alpha-1) J_{\text {Max }}^{2} \ell_{1}^{4} \leq \frac{1}{2} \frac{3+2 \alpha}{1+2 \alpha}+\frac{1+4 \alpha}{8(1+\alpha)^{2}} \pi^{2} .
$$

The only real difference with respect to Eq.(34) is the appearance of the linear $J_{\max }$ term. When the dominant energy condition is satisfied, we can replace $J_{\text {Max }}$ with $\rho_{\text {Max }}$ and get a quadratic expression in $\rho_{\text {Max }} \ell_{1}^{2}$. This in turn can be solved to give a direct bound on $\rho_{\operatorname{Max}} \ell_{1}^{2}$. When $\alpha=1$, this becomes

$$
\rho_{\mathrm{Max}} \ell_{1}^{2} \leq \frac{1}{8} \sqrt{\frac{301 \pi^{2}}{144}+\frac{5}{3}}-\frac{\pi}{6} \approx 0.07 .
$$

This is approximately three times smaller than the constant we obtained for the moment of time symmetry case in [6]. 


\section{MINIMAL SURFACES}

There is a very simple necessary condition for the existence of a minimal surface which is easy to derive and which is essentially gauge independent.. Let us return to the Hamiltonian constraint, Eq.(3). This can be rewritten as

$$
\frac{1}{2}\left(1+R^{\prime 2}\right)-\left(R R^{\prime}\right)^{\prime}=\frac{1}{4} R^{23} \mathcal{R}
$$

where ${ }^{3} \mathcal{R}$ is the three scalar curvature of the initial slice. If the weak energy condition is satisfied and if $0.5 \leq \alpha<\infty$ we have that ${ }^{3} \mathcal{R} \geq 0$. This is sufficient to show that $R^{\prime} \leq 1$. Let us assume that the initial data contains a minimal surface and that the first minimal surface occurs at $\ell=\ell_{M}$. Clearly, in the range $0 \leq \ell \leq \ell_{M}$, we have $0 \leq R^{\prime} \leq 1$. Let us integrate Eq.(58) from the origin out to $\ell_{M}$. We get

$$
\ell_{M} \geq \frac{1}{2} \int_{0}^{\ell_{M}}\left(1+R^{\prime 2}\right) d \ell=\frac{1}{16 \pi} \int_{0}^{\ell_{M}} 4 \pi R^{2}{ }^{3} \mathcal{R} d \ell=\frac{1}{16 \pi} \int_{0}^{\ell_{M}}{ }^{3} \mathcal{R} d v
$$

The boundary term can be discarded because $R^{\prime}=0$ at a minimal surface. Thus a necessary condition for the appearance of a minimal surface is

$$
16 \pi \ell \geq \int_{0}^{\ell_{M}} R^{(3)} d v
$$

If we have a minimal surface it must be either future or past trapped. Unfortunately, we cannot use this condition, Eq.(60), to derive a necessary condition for trapped surfaces because we could have a trapped surface without any minimal surface.

\section{CONCLUSIONS}

In this paper we have presented new necessary conditions for the presence of both apparent horizons and singularities in spherically symmetric initial data.

While we have assumed that spacetime is foliated extrinsically, this is not a severe restriction. Indeed, modulo the constraints, the destinction between intrinsic and extrinsic foliations becomes an artificial one.

The inequalities do not depend sensitively on $\alpha$. We have seen that just as one has to place a lower bound on $\alpha$ to obtain a sensible gauge, to obtain necessary conditions one needs also to impose an explicit upper bound on the spatial variation of $\alpha$. Acting as it does to mask the underlying physics, it is not at all surprising that the variation of $\alpha^{\prime}$ needs to bounded. It is, overall, surprising that all of the gauge ambiguity can be absorbed in such a simple way.

Our approach to functional analysis has been extremely heuristic - it is clear that some of the inequalities exploited in Sect. 4 and 5 can be sharpened, specially those relating to non-constant $\alpha$. As physicists, however, we always use the gauge which makes life easiest - linear gauges with $\alpha$ constant does this. When $\alpha$ is not constant, we are clearly more interested in the fact that such bounds can be established than in squeezing them for better constants. 
How is this work likely to be generalized? The obvious challenge is to generalize it to non-spherically symmetric geometries.

The Hoop conjecture formulated many years ago by Kip Thorne [8] states, in rough terms, that a black hole hole will form if and only if energy is compressed in all three spatial directions. If we admit 'cosmic censorship' the conjecture can be rephrased in terms of initial data, with black hole replaced by apparent horizon. It should be clear why the phrasing of the conjecture is vague. Even with no independent gravitational degrees of freedom to worry about, it is remarkably difficult to provide a description of the two ingredients 'quantity of matter' and size which is simultaneously valid for both necessity and sufficiency, never mind proving the conjecture. The situation can only get worse when we relax spherical symmetry. One needs to bear in mind that our ability to describe the configuration space in considerable detail has relied on features of the spherically symmetric problem which, we know, do not admit generalizations. Progress has been made on the sufficiency part of the conjecture [9]. Much less is known about the necessary part. Our work in this paper where the Poincaré ineqality on the interval plays a central role, suggests a new approach to attacking the problem in non-spherically symmetric geometries. This generalization might involve a Sobolev type inequality on the scale factor, $\Phi$ :

$$
S\left(\int d^{3} x \Phi^{6}\right)^{1 / 3} \leq \int d^{3} x(\nabla \Phi)^{2}
$$

Indeed, had we exploited conformal coordinates, with respect to which the spatial line element assumes the form, $d s^{2}=\Phi^{4} d s_{\text {Flat }}^{2}$, we would have found ourselves in need of such an inequality to derive the results of this paper.

We are encouraged by the fact that Sobelev inequalities are known to be related intimately with the isoperimetric problem [10].

A physically interesting question that is extremely relevant is the identification of initial data that potentially might develop apparent horizons. In principle it should be possible to do this exploiting in addition to the constraints, the dynamical Einstein equations evaluated on the initial hypersurface. These equations involve the pressure of matter though some equation of state. The scenario which is most susceptible to collapse is pressureless matter. We should be able to exploit this condition to formulate necessary conditions. At the other extreme, a stiff equation of state would inhibit collapse. Thus such a scenario might provide a sufficient condition.

\section{ACKNOWLEDGEMENTS}

We gratefully acknowledge support from CONACyT Grant 211085-5-0118PE to JG and Forbairt Grant SC/96/750 to NÓM.

\section{APPENDIX}

In this appendix, we provide a derivation of the bound for the the extrinsic curvature quadratic used in the text. 


$$
\int_{0}^{\ell_{1}} d \ell\left(\int_{0}^{\ell} d \ell R^{1+\alpha}\right)^{2} \leq\left(\frac{2}{\pi}\right)^{2} \ell_{1}^{2} \int_{0}^{\ell_{1}} d \ell R^{2(\alpha+1)} .
$$

The existence of a bound of this form is not hard to see. A crude bound is provided by the positivity of the covariance for any power $R^{n}$ :(Hölder Inequality),

$$
<R^{n}>^{2} \leq<R^{2 n}>
$$

which implies

$$
\left(\int_{0}^{\ell_{1}} d \ell R^{n}\right)^{2} \leq \ell_{1} \int_{0}^{\ell_{1}} d \ell R^{2 n}
$$

so that

$$
\int_{0}^{\ell_{1}} d \ell\left(\int_{0}^{\ell} d \ell R^{1+\alpha}\right)^{2} \leq \frac{\ell_{1}^{2}}{2} \int_{0}^{\ell_{1}} d \ell R^{2(1+\alpha)} .
$$

The bound (62) is, however, better. To derive it, let

$$
G(\ell):=\int_{0}^{\ell} d \ell R^{n}
$$

Now $G(0)=0$ and $G^{\prime}\left(\ell_{1}\right)=0$, for all $n \geq 0$. We apply the Poincaré inequality to $G$ with the constant which is appropriate with these boundary conditions:

$$
\int_{0}^{\ell_{1}} d \ell G_{n}(\ell)^{2} \leq\left(\frac{2 \ell_{1}}{\pi}\right)^{2} \int_{0}^{\ell_{1}} d \ell R^{2 n}
$$

so that

$$
\int_{0}^{\ell_{1}} d \ell\left(\int_{0}^{\ell} d \ell R^{(1+\alpha)}\right)^{2} \leq\left(\frac{2 \ell_{1}}{\pi}\right)^{2} \int_{0}^{\ell_{1}} d \ell R^{2(1+\alpha)} .
$$

This is better by a factor of $\pi^{2} / 8$ than the estimate (65).

The same bound is obtained for functions $R(\ell)$ satisfying Eq.(8) at $\ell=\ell_{1}$. The crude bound we derived before, (65), is expected to work better this time. As before, however, we can do better. This time we let

$$
H(\ell):=\int_{0}^{\ell} d \ell R^{n} / \int_{0}^{\ell_{1}} d \ell R^{n} .
$$

Now $H(0)=0$ and $H\left(\ell_{1}\right)=1$ for all $n$. We apply the Poincaré inequality to $H$ with the appropriate constant

$$
\int_{0}^{\ell_{1}} d \ell H(\ell)^{2} \leq \frac{1}{\gamma^{2}}\left(\frac{2 \ell_{1}}{\pi}\right)^{2} \int_{0}^{\ell_{1}} d \ell R^{2 n} /\left(\int_{0}^{\ell_{1}} d \ell R^{n}\right)^{2}
$$

where $\gamma$ is given by Eq.(49). Exploiting the lower bound on $\gamma$ obtained in the text we obtain Eq. (62) exactly as we did for singularities. 


\section{REFERENCES}

[1] J. Guven and N. Ó Murchadha, Sufficient Conditions for Apparent Horizons in Spherically Symmetric Initial Data (1997)

[2] [四] extends earlier work by P. Bizoń, E. Malec and N. Ó Murchadha, Phys. Rev. Lett. 61, 1147 (1988); Class Quantum Grav 6, 961 (1989); 7, 1953 (1990) as well as [5] where the problem is addressed for maximally sliced spherically symmetric geometries to arbitrary extrinsic time foliations.

[3] We exploit the notation introduced in J. Guven and N. Ó Murchadha, Phys Rev D52 758 (1995) An extensive list of references is provided here.

[4] J. Guven and N. Ó Murchadha, Geometric Bounds in Spherically Symmetric General Relativity (1997)

[5] E. Malec and N. Ó Murchadha Phys. Rev. D50 R6033 (1994)

[6] J. Guven and N. Ó Murchadha, Phys Rev D52 776 (1995)

[7] In [6], in our examination of a moment of time symmetry, a weaker statement was formulated: if $M<\ell / 2$ on a given surface then that surface is not trapped. If $J \neq 0$, however, even a statement of this form is beyond our reach. The reader is referred to 66] for details.

[8] K. Thorne, in Magic without Magic: John Archibald Wheeler, edited by J. Klauder (Freeman, San Francisco, 1972)

[9] For a partial list see, for example, R. Schoen and S.T. Yau, Comm. Math. Phys. 90, 575 (1983); R. Beig and N. Ó Murchadha, Phys. Rev. Lett. 66, 2421 (1991); E. Malec, Phys. Rev. Lett. 67, 949 (1991); Mod. Phys. Lett. A7, 1679 (1993); T. Zannias, Phys. Rev. D47, 1448 (1993); E. Flannagan, Phys. Rev. D44, 2409 (1991); D46, 1429 (1992); D. Eardley, J. Math. Phys. 36, 3004 (1995); G. Burnett, J. Math. Phys. 37, 1434 (1996) G. Gibbons, hep-th/9701049

[10] See, for example, I. Chavel, Eigenvalues in Riemannian Geometry (Academic Press, New York, 1984) 